\title{
Direct Electron Detection for Atomic-Resolution EELS Mapping at Cryogenic Temperature
}

David J. Baek ${ }^{1}$, Michael J. Zachman², Berit H. Goodge ${ }^{2}$, Di Lu ${ }^{3}$, Yasuyuki Hikita ${ }^{4}$, Harold Y. Hwang ${ }^{3,4}$, and Lena F. Kourkoutis ${ }^{2,5}$

1. School of Electrical and Computer Engineering, Cornell University, Ithaca, NY, USA.

2. School of Applied and Engineering Physics, Cornell University, Ithaca, NY, USA.

3. Department of Applied Physics, Stanford University, Stanford, CA, USA.

4. Stanford Institute for Materials and Energy Sciences, SLAC National Accelerator Laboratory, Menlo Park, CA, USA.

5. Kavli Institute at Cornell for Nanoscale Science, Cornell University, Ithaca, NY, USA.

Technical advances in spectroscopic imaging using electron energy loss spectroscopy (EELS) have allowed a material's chemical and electronic structure to be resolved at the atomic scale [1]. While this can now be achieved almost routinely for radiation-hard materials studied at room temperature (RT), cryogenic applications are hampered by the reduced stability of side-entry cryo-holders. In these holders, the sample stage is in direct thermal contact with a liquid nitrogen $\left(\mathrm{LN}_{2}\right)$ bath, resulting in increased stage drift and instabilities due to cryogen bubbling. While bubbling can be almost eliminated, increased sample drift requires rapid data collection. This is particularly challenging for spectroscopic mapping as it requires pixel dwell times that are orders of magnitude larger than typical imaging dwell times. Low temperature EELS experiments have, therefore, focused on collecting area-averaged spectra or line profiles [2, 3]. However, to understand spatial modulations and nanoscale inhomogeneities of electronic states, atomically-resolved spectroscopic imaging at low temperatures is required. More efficient detectors hold great promise for cryo-EELS. Here, using a direct electron detector (DED) with improved detector quantum efficiency, point spread function, and signal-to-noise ratio compared to indirect, scintillator-based detectors [4], we demonstrate atom-resolved 2-D EELS mapping of a charge ordered (CO) manganite at $\mathrm{LN}_{2}$ temperature.

$\mathrm{Nd}_{0.5} \mathrm{Sr}_{0.5} \mathrm{MnO}_{3}$ (NSMO) is a paramagnetic insulator at RT. Upon cooling it undergoes a phase transition to a ferromagnetic metal at $\sim 250 \mathrm{~K}$ followed by a transition into a $\mathrm{CO}$ insulating state at $\sim 160 \mathrm{~K}$. We perform cryogenic EELS mapping near $\mathrm{LN}_{2}$ temperature to directly visualize the $\mathrm{CO}$ phase. Using a Gatan K2 Summit DED enabled for spectroscopy, a beam current of 150pA and a short dwell time of $2.5 \mathrm{msec} /$ pixel are used to record a $70 \times 70$ pixels 2-D map in only 13 seconds. Such rapid mapping is enabled by the DED's reduced read out noise and dead time and provides atomic-resolution maps which include fine-structure information to be obtained at $\sim 96 \mathrm{~K}$ (Figure 1). To extract bonding variations, multivariate curve resolution is performed on the $\mathrm{Mn}-\mathrm{L}$ and $\mathrm{O}-\mathrm{K}$ edges, which demonstrates electronic structural differences between the RT state and the low temperature CO state in NSMO (Figure 2). Enhanced detector sensitivity in EELS is expected to play a larger role for applications that require not only rapid data acquisition, but also less electron dose [5].

\section{References:}

[1] D. A. Muller et al, Science 319 (2008), p. 1073.

[2] R. F. Klie et al, Phys. Rev. Lett. 99 (2007), p. 047203.

[3] W. Zhao et al, 2017, arXiv:1701.03678. 
[4] J. L. Hart et al, Scientific Reports 7 (2017), p. 8243.

[5] This work was supported by the Department of Defense Air Force Office of Scientific Research (FA 9550-16-1-0305) and National Science Foundation (DMR-1539918, DMR-1429155, DMR-1719875).

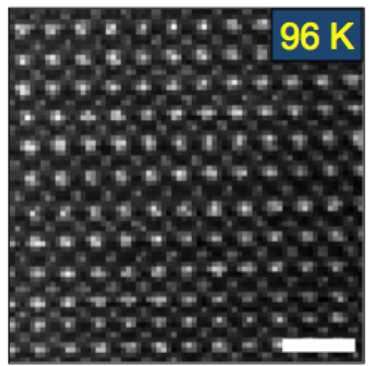

Simultaneous ADF

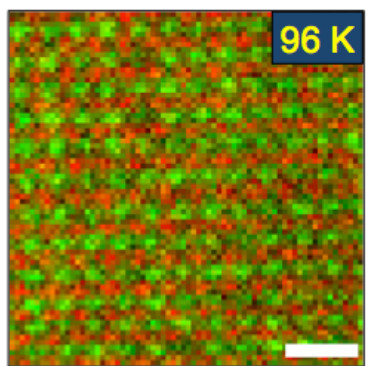

False colormap: $\mathrm{Nd} \mathrm{Mn}$

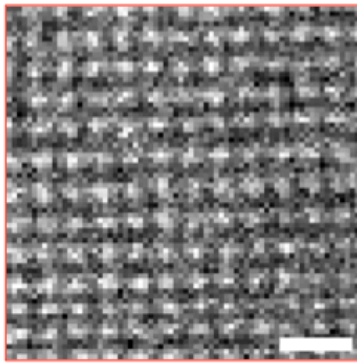

$\mathrm{Mn}-\mathrm{L}_{2,3}$

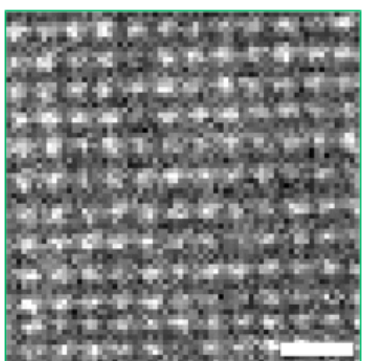

$\mathrm{Nd}-\mathrm{M}_{4,5}$
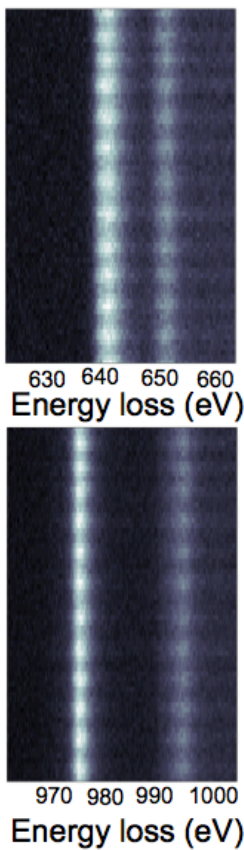

Figure 1. Left column: Simultaneously recorded ADF image (top) and false colored image of $\mathrm{Nd}$ and Mn (bottom) acquired at 96K. Middle column: Elemental maps extracted from $\mathrm{Mn}-\mathrm{L}_{2,3}$ (top) and $\mathrm{Nd}-$ $\mathrm{M}_{4,5}$ (bottom) edges at 96K. Right column: Background-subtracted Mn-L2,3 (top) and Nd-M4,5 (bottom) spectra summed laterally, showing fine structures resolved with atomic-resolution. (Scale bars: $1 \mathrm{~nm}$ )
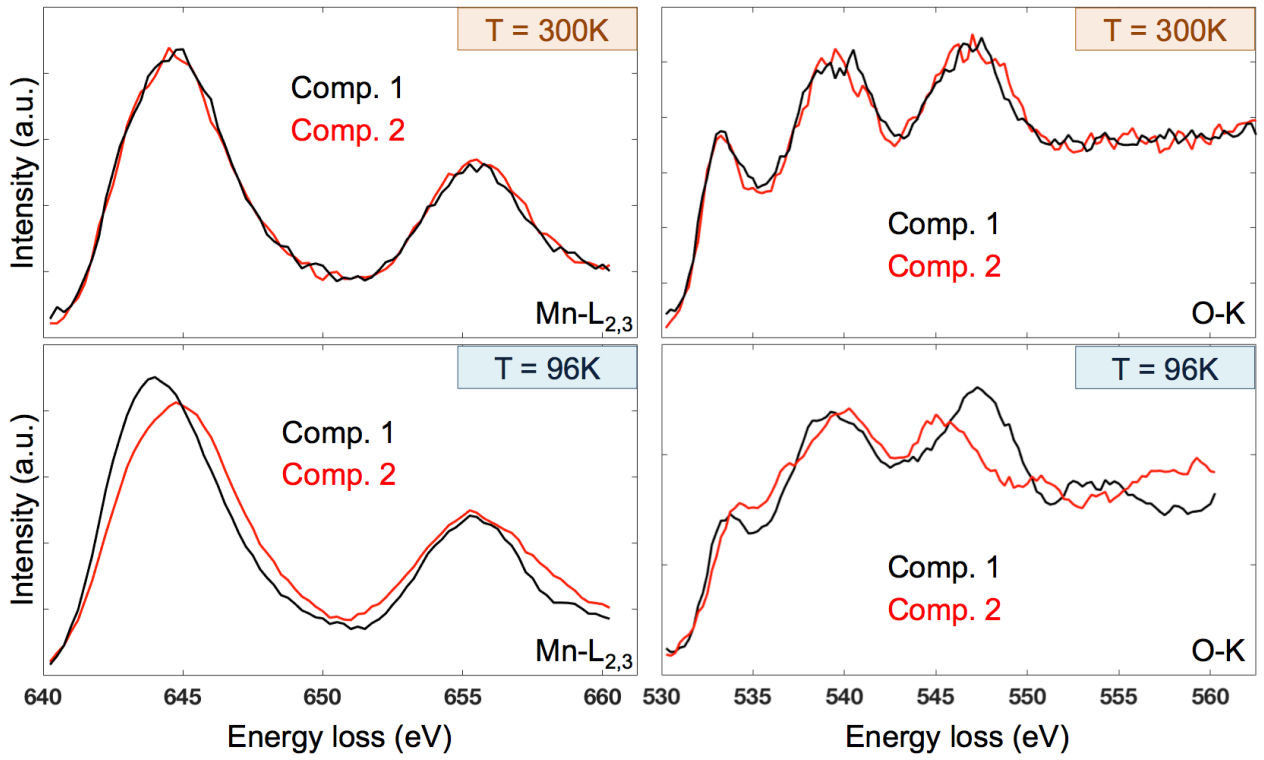

Figure 2. EELS spectra of $\mathrm{Mn}-\mathrm{L}_{2,3}$ and $\mathrm{O}-\mathrm{K}$ edges acquired at room and cryogenic temperatures from a $\mathrm{Nd}_{0.5} \mathrm{Sr}_{0.5} \mathrm{MnO}_{3}$ thin film. From an EELS map obtained at $96 \mathrm{~K}$, two distinct components are extracted for both edges using multivariate curve resolution. At 300K, however, only a single component exists. 